\title{
The Internet of Things and People in Health Care
}

\author{
Nancy L. Russo and Jeanette Eriksson \\ Department of Computer Science and Media Technology, Internet of Things and People \\ (IoTaP) Research Center, Malmö University, Malmö, Sweden
}

\subsection{Introduction}

Technology has long been viewed as a means of improving health care delivery, ranging from the ability to store and access digital health records to more complex applications such as using artificial intelligence to diagnose disease or using robots to perform surgery. Technology can help overcome limitations in resources (financial and personnel), can reach patients living long distances from health care specialists, and can help communities deal with aging populations. Both the United Nations and the World Health Organization have identified the need and potential for technology to support the delivery of health care services to underserved populations (UN, 2010; WHO, 2010). The ability to capture, store, analyze, and disseminate health information using technology can reduce the human resources needed to manage this process and improve the accuracy of the data. The ability to perform rapid analysis on this health data can ensure that scarce health care resources are allocated where needed most urgently. In remote, rural areas of many parts of the world, health care providers and services are often not available locally, requiring patients to travel long distances to obtain care. Technology, such as mobile device-based videoconferencing, can allow patients to be diagnosed and potentially treated remotely by doctors and other health care professionals. Technology can support communities faced with caring for growing numbers of elderly patients by providing the ability to monitor patients' vital signs, to easily track patients' eating, exercise, and medication schedules and provide reminders, and to strengthen social engagement between elderly patients and other community members. All of these services facilitated by technology can reduce the demands on health care professionals and other caregivers and increase the quality of life for patients.

Internet of Things A to Z: Technologies and Applications, First Edition. Edited by Qusay F. Hassan. 
The Internet of Things (IoT) expands the potential for using technology to support health care by connecting not only people, applications, and data, but also sensors and devices that collect biometric and contextual data. These health care ecosystems may include smart devices that are capable of using this data to take actions, such as providing alerts or sending notifications, when a measured value reaches a certain target level, providing recommendations to patients or health care professionals based on analysis of data, and potentially delivering medication based on monitored patient data and medical guidelines.

IoT-based systems can be used to address a broad set of health issues ranging from well-being to sickness, physical to mental health, preventive care to treatment or rehabilitation, and temporary disabilities to chronic disease. Smartphones, watches, and other smart devices as well as additional sensors, devices, and equipment can be connected to IoT networks to provide information about the patient, the patient's activities, and the patient's context or environment. By using IoT technologies to capture physiological measures and kinesiological data as well as other context data, smart health systems can empower people to proactively engage in their health as well as to manage their recovery from illness or injury. In addition, smart health can be used in health care settings to involve patients in their treatment and to share data about things such as treatment effectiveness. The patient, health care providers and caregivers, and other stakeholders, such as the patient's family members and friends, can also be part of the IoT ecosystem. This integration of human and technological agents and the sharing of vast amounts of data made possible by the Internet of Things, along with the applications that provide sophisticated data analysis, support better decisions about the patient's health and ultimately better health outcomes.

The focus of this chapter is IoT applications that support patients' health. These can be part of the broader health care system but are mainly used outside the hospital setting to collect and make use of data during the patient's daily life. Health care systems that are used solely in clinical, surgical, or laboratory settings are not discussed in this chapter, nor are health care systems that are primarily administrative or logistical in nature.

\subsection{The Smart Health Care Ecosystem}

The model in Figure 16.1 provides an overview of the components that may be included in an IoT-based smart health ecosystem. This is called an ecosystem to represent the complex and interconnected nature of the relationships between the human participants, the autonomous and subordinate devices, and the applications, interfaces, and data. This type of system or ecosystem is different from the early e-health systems that enabled a patient's health care data to be stored digitally and accessed remotely via the Internet and mobile devices (often 


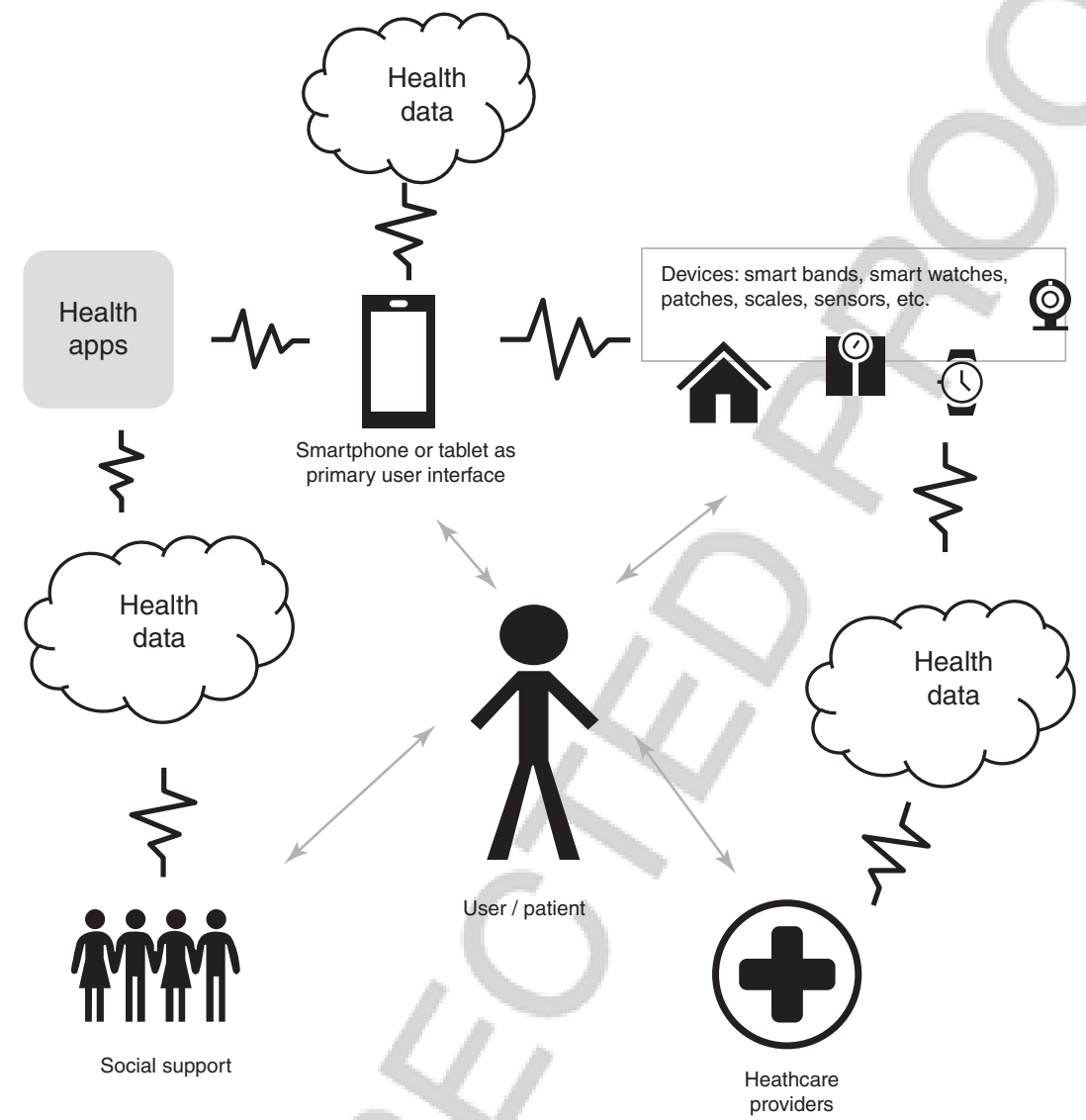

Figure 16.1 Smart health care ecosystem.

called m-health), or the ability to have an online consultation with a physician (telehealth). Now via the IoT, connected devices also store, access, and share data with other components of the ecosystem. Acting alone or in conjunction with other components, some nonhuman members (smart devices or agents) of the ecosystem have the capability to make decisions and take actions; for this reason, the ecosystem is considered to be "smart."

\subsubsection{The Patient at the Center}

The patient is illustrated at the center of this ecosystem because the primary purpose of a smart health care ecosystem is to support the patient in understanding, monitoring, and managing a health care-related issue. Trends in consumer use of applications and websites show increasing use of apps and 
websites to obtain information about health, to monitor health, and to more quickly interact with health care providers. This is driven both by the consumers increased health awareness and increasing familiarity in using computer-based products on a daily basis (Glaros and Fotiadis, 2005). People are both asked and enabled to play a more active role in their care. In addition, global economic challenges have led to reductions in resources for health care, which results in health care professionals having less time to spend with each patient, thereby limiting the opportunity to answer questions or discuss treatments. Patients spend less time in the hospital and more time recuperating on an outpatient basis. Therefore, patients have a greater need to obtain information related to their own health and have to take a more active role in managing all aspects of their health, from basic fitness and well-being to recovery from illness or injury as well as managing chronic diseases. Through the IoT, patients can be connected easily to other components of the ecosystem, including health care providers, a social support network, different types of devices and sensors, and applications running both locally and remotely, and can have access to data and services via familiar interfaces on smartphones, tablets, and computers. Each of these components will be described in more detail below.

\subsubsection{Health Care Providers}

The health care providers interacting with an IoT-based health care system include physicians and other medical specialists, pharmacists, nurses, therapists (such as physical, behavioral, or occupational therapists), laboratory technicians, and other caregivers. Health care providers interact with a smart health care system in a number of different ways, such as monitoring, diagnosing, treating, recommending actions, or communicating with the patient, the patient's family members, or with other health care providers.

A smart health care ecosystem provides the health care providers with access to continuous measures of patients' conditions, possibly on a number of metrics, over time. In addition, sophisticated diagnostic tools (applications and devices) may also be part of the ecosystem. Compared to what is possible with a patient's periodic visits to the physician's office or checkups from a visiting nurse, for example, this volume of data can allow the health care provider to recognize issues more quickly and to respond more rapidly to problems as well as to direct interventions to prevent more serious consequences. Medical professionals can more directly manage a patient's care by changing recommended treatments or medication levels based on analysis of the patent's activities, physiological measures, and other data. In managing patients' health-related behaviors, such as smoking cessation or weight reduction, health care providers may communicate with patients to set goals, monitor adherence, and provide feedback and recommendations to assist the patient in changing behavior. 


\subsubsection{Devices and Sensors}

The number of devices and sensors that can be connected to the IoT is growing rapidly. Some devices and sensors are connected to the patient to measure heart activity (ECG), electric currents in the brain (EEG), retina activity (EOG), emotional stress through galvanic skin response (GSR), blood pressure, blood oxygen levels, and heart rate. Many of these physiological metrics can now be measured via wearable sensors that may be incorporated in a wristwatch-type device or sensors that are built into smartphones. Wearable devices may also track location (via GPS), sleep patterns, and activity levels, typically via number of steps. Gyroscopes and accelerometers in wearable devices measure orientation and speed. Other types of wearables include a vest that measures heart rhythms and can perform defibrillation when abnormal rhythms are detected and booties that monitor babies' heart and breathing rates. Other devices and sensors connected to the IoT include household appliances that can share data, and sensors that detect motion, air quality, temperature, and humidity. Devices such as scales, cameras, blood pressure monitors, eye trackers, glucose monitors, and insulin pumps can be connected. Even specialized clothing and furniture can contain IoT-connected sensors that monitor physiological and kinesiological measures, such as a smart hospital bed that tracks a patient's vital signs. Any device that can produce or receive data can potentially become part of the health care IoT ecosystem.

\subsubsection{Applications and Interfaces}

To make the data captured by IoT-connected devices useful, applications are needed to process the data and present it to users (whether humans, applications, or other devices) in a meaningful way using well-designed interfaces. Most patients, health care providers, and other stakeholders interact with health care applications via a smartphone, tablet, or computer. Although the most common interfaces are visual (data is displayed on a screen and users interact with the screen directly or through a keyboard and mouse) interactions may also be verbal, motion driven, haptic, or directly from device to device or application to application. Some application interfaces provide a standard set of raw metrics with no interpretation or analysis and no customization features. Other application interfaces provide data that are customized for the particular users, and others allow users to personalize the method of reporting, or even of collecting, data. The application may compare the data to that of a representative population or to medically accepted standards.

Applications allow devices connected to the smart health care ecosystem to have different levels of autonomy. Some applications are passive, in that they record and report data but do not take any actions based on that data. Autonomous applications may be programmed to send alerts when particular 
thresholds are breached, to take action by itself, or even to direct another device to take some action. Section 16.4 will discuss examples of health care IoT applications.

\subsubsection{Other Stakeholders: Social Support}

The model of the smart health care ecosystem shows three types of human participants: the patient, the health care providers, and the social support. The individuals included in social support could be family members, friends, coworkers, and members of other social networks to which the patient belongs such as patient support groups. It is also possible that social workers, residence managers, drivers, and other service providers could be included as well.

Many health care situations could benefit from the ability to notify some member of a patient's social support network. For example, if a child's glucose level drops below acceptable levels during the night, an alarm could wake a parent, or if a patient being monitored for depression has not showered in several days (determined by a sensor that measures humidity levels in the bathroom), a friend or family member could be prompted to check on the patient. Patients using a behavior modification application to attempt to lose weight could elect to notify friends and family of their weight loss goal and keep them notified about progress. Some studies have shown that telling others about a goal and receiving the support of friends and family contribute to more success.

\subsubsection{Connecting the Components}

To enable the sharing of data that underlies the IoT, various devices, sensors, computers, and applications must be connected. In most instances the connection is wireless, but it is not always via the Internet. Connectivity may also be provided by cellular networks ( $2 \mathrm{G}-5 \mathrm{G}$ or LTE), by Wide Area Networks, or using Bluetooth or NFC (near-field communication) protocols. In some systems where relatively low amounts of data must be transmitted over short ranges, WPAN (wireless personal area networks), such as ZigBee, provide a communication channel that is very efficient in terms of power consumption. An IoT health care ecosystem may utilize multiple communication channels.

\subsubsection{Summing up Smart Health Care Ecosystem}

As an example that illustrates how all components of the ecosystem interact, assume that a patient is recuperating from surgery to repair a broken hip. The patient wears a device on his wrist that monitors activity (such as walking) and can sense when the patient has fallen, and a sensor in his shoe measures gait and walking speed. Outputs from these devices are stored in the cloud. An application monitors this data and makes it available for a health care provider to 
check periodically. If the patient has not walked the number of steps "prescribed" by the health care provider, he is sent a reminder via his mobile phone. If the patient's gait and walking speed indicate poor balance, the health care provider is notified so that some intervention may be taken, such as scheduling an appointment with a physical therapist or a checkup with a visiting nurse. If a fall is detected, the patient's family member or other local caregiver is sent an urgent notification via SMS and the health care provider is also notified. It is also possible that emergency services could be contacted. All of the data on these events would be available to the health care provider, along with other data such as medication history, to use when doing periodic assessment of patient treatment plans.

Not every smart health system involves all of the components. All, however, use the IoT as the foundation for sharing data. Although the ecosystem identifies the basic building blocks of IoT systems for health care, it is necessary to look deeper at the applications to explore the many different ways the IoT can be used in patient-centered health care contexts. The following section will describe IoT-supported smart health care applications by evaluating the problem and solution space covered by the applications in terms of seven different characteristics called dimensions.

\subsection{Dimensions of Internet of Things Applications in Health Care}

As already discussed, a breadth of contexts are currently addressed by smart health care applications that focus on the patient. These health care applications can be described by how they address aspects of the health care situation:

1) The Type of Health Care Issue. General maintenance of health or well-being or a particular illness or injury or other health care event.

2) The Locus of the Health Care Issue. Physical (of the body) or mental (related to functioning of the mind).

3) The Time Dimension. One-time or temporary versus on-going or chronic.

4) The Goal of the Application. To prevent a condition, or to prevent a condition from getting worse, or to treat a condition with the goal of curing it.

5) The Degree of Action Provided by the Application. Monitoring data about the situation, or providing data to allow users to monitor the data, versus taking action or supporting the user or other stakeholder in taking action to manage the situation.

6) The Source of Data. Internal measures related to the condition of the patient (generated by the patient) or measures external to the patient but relevant to the health care situation.

7) The Primary User of the Application. Used on an individual level by the patient for self-care, or by a patient's family member(s) for monitoring the 
patient versus applications used by health care providers or other caregivers to monitor or assist the patient or to evaluate treatments. It is assumed that informal social support could be used with any application and thus it is not shown as a separate dimension.

To make a visual representation of a smart health care application, the dimensions can be combined into one diagram (Figure 16.2). On this diagram, which resembles a snowflake, it is possible to indicate how strongly the individual dimensions are represented in the application. Each dimension represents a range or continuum between one end-point value and the other. Particular applications could fall anywhere along the range, but because this positioning is not an absolute value there are simply two states shown: some or all. Therefore, a dimension that is partially or somewhat addressed in an application would be marked up to the first ' $X$ ' on the dimension on the snowflake. A dimension that is identified as the focus of an application, and thus more fully addressed by the application, would be marked to the ' $\mathrm{X}$ ' at the end of the dimension range. This identification of the degree to which applications address the dimensions allows categorization and comparisons of different applications on the various dimensions. Each of these seven dimensions is described in more detail below.

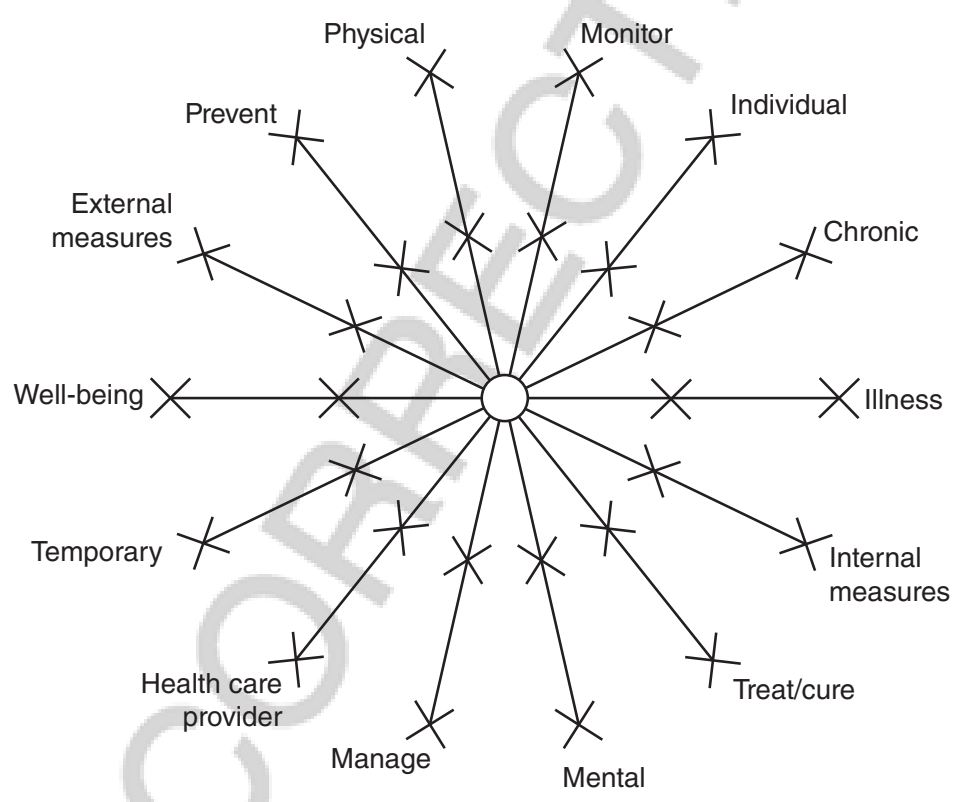

Figure 16.2 A snowflake model representing the seven dimensions of smart health care applications. (Note: If the dimension is only partially addressed by an application, the appropriate end of the dimension line will be marked with a bold line to the first " $\mathrm{X}$ " (from the center); if the dimension is a major aspect of the application, the entire line relating to that end of the dimension will be marked with a bold line.) 
To relate this model to the smart health ecosystem model in Figure 16.1, it should be noted that the focus of this chapter has been patient-centered systems, so all applications of the model involve data about the patient. Several of the dimensions describe aspects of the data: its source, its primary user, and the type of health care issues to which it is applied. The social support component of the ecosystem is not shown as a separate dimension because the output of any application could potentially be shared with others via social media (and at times directly from the application itself), and the individuals providing social support, while important, are secondary to the patient and the health care providers in terms of managing the patient's health.

\subsubsection{Well-being-IIlness Dimension}

The quantified self movement represents an extreme manifestation of selftracking for the purpose of well-being or health maintenance. It is common today for smartphone applications and fitness tracking bands to automatically track metrics such as steps, sleep, and heart rate. Some applications can also measure stress level and other types of exercise. Many applications allow users to enter information on food consumption, emotional status, weight, and any other metrics the user might want to track. Because these applications are connected to the IoT, they can also exchange information with other devices such as smart scales. These types of applications would for the most part be considered to address the user's general health and fitness. Performance improvement applications, such as those used by runners to track their speed and endurance, would also fall into this category.

At the other end of this dimension, there are applications that address a specific illness or condition such as strep throat, diabetes, asthma, or COPD (chronic obstructive pulmonary disease), injury such as a broken bone or dislocated kneecap, or other health incidents such as stroke. Applications that address a particular health issue may use specialized equipment as well as many of the same sensors used for wellness applications, but in conjunction with specialized programs to analyze the metrics and determine the appropriate response.

\subsubsection{Physical-Mental Dimension}

Health care can be separated into the two broad categories of physical health and mental health. Physical health includes issues related to the body. Physical health issues could be related to functioning of the various systems (respiratory, vascular, etc.) or to organs or to communications between them; to bones, muscles, and connective tissue and their functioning; and to the ability of the individual to perform tasks related to living, work, and leisure. Mental health encompasses intellectual, cognitive, and emotional health. Conditions related to 
mental illness, such as depression, schizophrenia, and obsessive-compulsive disorder, as well as addiction, anger management issues, and cognitive impairments, such as memory loss, would be classified as mental health issues.

\subsubsection{Temporary-Chronic Dimension}

Some health-related issues are temporary in nature-a broken bone has to heal or medication must be taken for a certain number of days to vanquish an infection. Other health-related issues are chronic because they exist over a long period of time, perhaps a lifetime. While it can be assumed that temporary health-related issues are those that will be dealt with within a fixed amount of time, it is of course possible that the particular amount of time may not be easily determined. Certain issues, such as losing a particular amount of body weight, could be considered temporary yet may require lifelong monitoring to maintain. Therefore, weight loss applications would be categorized as temporary while weight maintenance applications would be categorized as addressing a chronic condition.

\subsubsection{Prevent-Cure Dimension}

Smart health applications that allow users to monitor their weight, food intake, and activity levels can be viewed as applications that could be used with the goal of preventing the onset of a disease such as type 2 diabetes. Applications may also be used to aid in the prevention of serious complications from an illness, such as asthma attacks or uncontrolled bleeding due to lack of coagulants in the blood. Other IoT-based health systems can be used in curing diseases or supporting recovery from an injury, illness, or other health event. Applications that monitor adherence to prescribed medication regimes can increase the likelihood of successful treatment of malaria, tuberculosis, or a bladder infection. IoT systems can aid in the rehabilitation of individuals who are recovering from surgery or have suffered a stroke or a broken bone, for example, and need to retrain muscles or to regain strength and endurance through recommended exercises. Applications can provide information on what movements or exercises should be performed (and those that should be avoided) and can track whether or not they have been performed either autonomously via sensors or via a user's self-reported data. In addition to prompting the patient to perform the exercises or take the prescribed medication, these applications may also be able to inform health care providers and members of the patient's social support network if the patient is not adhering to the care plan.

\subsubsection{Monitor-Manage Dimension}

IoT systems may be used to both monitor and actively manage health-related conditions. Monitoring systems provide access to data for the patient, health 
care professionals, and/or other caregivers. While some systems are strictly for personal monitoring, others track data and compare it to a larger population or to previous results for the individual (or both) and allow the sharing of data with others. Personal monitoring systems assist patients in understanding the impact of different activities or situations on their health. Some monitoring systems allow health care professionals and other caregivers to monitor particular metrics remotely. This may be to enable health care professionals to assess treatment effectiveness over many different patients over time, or to monitor whether a patient is progressing in their rehabilitation. In other applications, the intention may be to allow faster interventions by the health care professional if a patient's measures go outside desired ranges.

Applications that manage health care issues are more likely to include interaction with health care professionals, although this is not always the case. Applications that help patients manage depression or anxiety may include active participation by therapists or psychiatrists, particularly if the illness is severe and medication is used. As an extreme example of patients directly managing their own health care situation, some type 1 diabetes patients frustrated with the slow pace of the medical establishment's introduction of diabetes management systems that both track glucose levels and administer insulin have circumvented the medical professionals and regulatory bodies and built their own systems. These Artificial Pancreas Systems link a patient's continuous glucose monitoring device with an insulin pump via the IoT using an inexpensive computer (the Raspberry $\mathrm{Pi}$ ). Instructions for programming the application are provided online ${ }^{1}$. While the system still requires patients to monitor their glucose levels and make the final determination about insulin dosage, the system can administer the dosage once approved by the patient.

\subsubsection{Internal-External Measures Dimension}

Some health-related systems rely exclusively on data that come from measures of the individual, often generated by sensors, in smartphones or wearables, or other devices that measure functions of the individual user. This data may be used directly in its raw form or it may be used as input to more complex calculations. Examples of physiological measures that come directly from measurements of the patient include heart rate, number of steps, body temperature, weight, pupil size, brain activity (EEG), and glucose level. Other data relevant to a health care-related application may come from the context of the individual user's environment, such as data about location, humidity, temperature, and altitude or about events on the user's calendar, other applications they have used, social media access, and phone usage. Other data commonly used in health care applications include patient's self-reported data such as food

1 https://openaps.org/ 
consumption, sleep quality, energy level, mood, stress level, and other information specifically related to the application focus. To determine whether to categorize data as internal or external, the locus of the generation of the data is considered. If it is generated by the patient's body, it is considered internal. If the data is generated outside of the patient's body, it is considered external.

\subsubsection{Health Care Provider-Individual Dimension}

This final dimension addresses the primary user of the health-related application (particularly the data). Many applications are used primarily by the patient to monitor or manage health-related issues. At this end of the spectrum, patients would provide data to the system via sensors, devices, or self-reporting, and patients would also be the primary recipients of the data to use in their own selfcare. At the other end of the spectrum would be applications that are used primarily by health care providers (doctors, nurses, and other specialists) to monitor patients or to acquire information to use in evaluating treatment plans. While it is possible that some health-care-provider-focused applications that collect data from the patients via sensors, devices, or self-reporting would provide no data to the patient, it is more likely that the patient will be able to see the data, although the patient is not the targeted recipient. Most smart health care applications provide data to both the patient and to the health care provider. When data is shared with family members, friends, or other social support network members, this would be considered an extension of the individual dimension.

\subsection{Examples of loT-Related Health Care Applications and Their Dimensions}

It would be impossible to identify all of the smart health care applications under development or available today, so this section will describe a sample of these applications. The focus is on applications that focus on the patient, rather than on applications that support administrative or logistical functions such as tracking the locations of medical equipment within a hospital. There is sometimes a misperception that all patient-focused IoT applications are smartphone apps that use the Internet to share data. Although these are quite common, they are not the only types of patient-focused IoT health care applications. Some systems use specialized output to other devices, such as an infusion pump to deliver medication. Data may be shared over Bluetooth or RFID networks, for example. In this section, some of the diversity found in IoT health care applications will be illustrated through examples of several products that are currently in use or in the late stages of testing in preparation for deployment. These examples are provided for educational purposes, and the discussion is not 


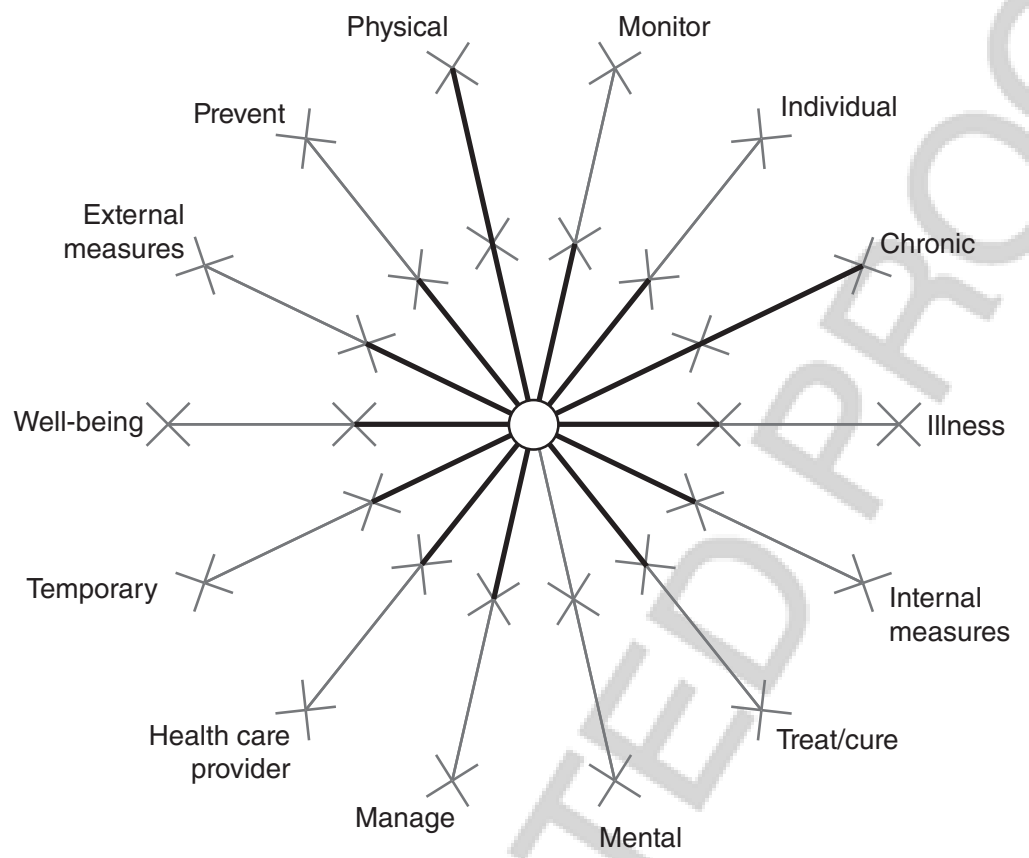

Figure 16.3 EpiWatch represented in the snowflake model.

intended to serve as endorsement for the products. A website (active at the time of publication) is provided for each of these applications. Each application will be described using the snowflake model of health care application dimensions that was introduced in the previous section. The extent to which the application addresses a particular dimension is indicated using a bold line: a bold line going halfway indicates that the application only partially addresses that aspect, and a bold line fully covering one end of the dimension indicates that the particular aspect is a central part of the application. In the text description of the applications, italics are used to highlight the particular aspects of each dimension addressed by the application.

EpiWatch ${ }^{2}$ is an Apple Watch application that enables users who have epilepsy to track seizures, daily medication use, and possible medication interactions, and to share this information with medical researchers. This data, in conjunction with an accelerometer and a heart rate monitor, may ultimately be able to predict seizures. In Figure 16.3, the dimensions of the EpiWatch are shown in the snowflake model.

The EpiWatch is designed for a physical chronic disease, epilepsy. Epilepsy may be caused by a brain injury or stroke, or may be a genetic disorder. In either

2 www.hopkinsmedicine.org/epiwatch 
case, the result is abnormal functioning of brain cell activity that leads to seizures. If a person gets epilepsy before the age of ten the disease is often temporary, as a child's brain may heal and return to normal functioning, but many people have to live with the disease throughout their life. The EpiWatch app makes it possible for individual patients to monitor their seizures and the monitoring makes it possible for researchers and health care providers to better understand epilepsy for better treatment, and also in the future to be able to detect and prevent seizures. Epilepsy is a kind of illness in the brain, but the objective of the EpiWatch is to make it possible for people to live a good life without worrying about their next seizure, thus for their well-being rather than solely for treating the illness. The sensors in the watch measure internal measurements, that is, data automatically generated by the user's body, as it measures heart rate and the user's movements while having a seizure. The app also collects external data as the users can input data about their quality of life.

The Owlet Smart Sock $2^{3}$ is a baby bootie that uses pulse oximetry to measure an infant's heart rate and oxygen levels while they sleep. The sock, or bootie, is worn on the infant's foot and is held in place by a Velcro strap. The sensor is embedded into the sock. The heart rate and oxygen level data captured by the sensor can be viewed on a smartphone via the app. If heart rate or oxygen levels go outside of predetermined bounds, an alarm uses light and sound to notify a parent. In Figure 16.4 the dimensions of the Smart Sock 2 are presented in the snowflake model.

The Smart Sock is designed for babies and their parents. It is designed for individuals and health care providers are not involved at all. The socks are entirely designed for the baby's and the parents' well-being by preventing the physical affliction, early infant death (also called SIDS or sudden infant death syndrome), by alerting parents so they can take action. The Smart Sock is not curing or treating the affliction. By using Smart Sock the parents can monitor their newborn baby and feel safe that the sock will send an alarm if the baby's breathing deviates from the normal. The monitoring is just temporarily needed as the risk for early infant death will decrease over time and eventually the risk will fade. The sensors in the sock collect internal data like heart rate and oxygen in the blood.

Propeller ${ }^{4}$ is an inhaler used by patients with asthma or chronic obstructive pulmonary disease (COPD). For both of these, patients often use an inhaler to get medication into the lungs to facilitate breathing. With Propeller, the user connects a sensor to his or her inhaler and uses the medication as usual. The sensor records when the inhaler was used. Other data captured by the application (such as location, time of day, air quality) allow the user to gain insight into triggers for breathing difficulty. The application can provide results to family and

3 https://www.owletcare.com/

4 www.propellerhealth.com 


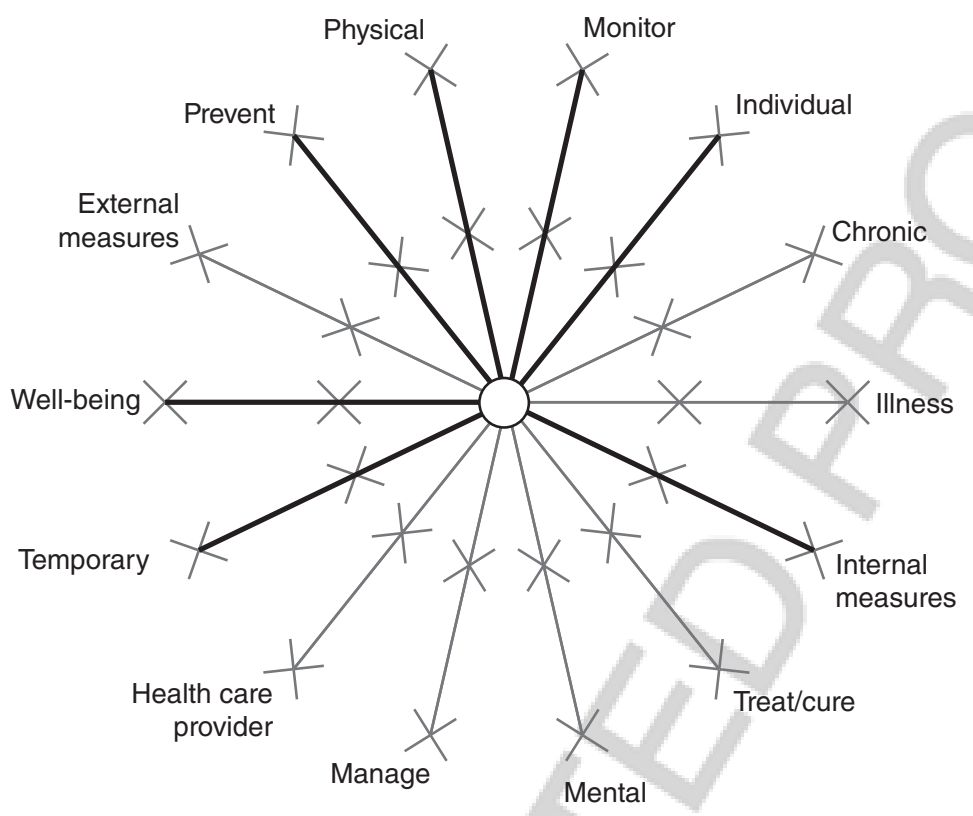

Figure 16.4 Smart Sock 2 represented in the snowflake model.

health care providers. In Figure 16.5 the dimensions of the Propeller device are mapped out in the snowflake model.

Asthma and COPD are physical illnesses. Most of the people suffering from asthma have a less severe form, but some patients have chronic asthma with seizures. COPD is also something you have to live with throughout your life. The device is only collecting external data such as air pollution and context data of the inhaler use. The application can inform the user of the air quality and the user can thereby prevent a seizure by taking medicine or avoiding the polluted area. In this way, it is possible for the user to not only monitor the environment and the medicine intake, but also makes it possible for the user to manage the disease by learning what triggers a seizure and in this way have a freer and better quality of life, thus increasing their well-being. The device, of course, benefits the user, but the data is also available for the health care providers that can improve the relationship between the individual and the health care provider as they both share the knowledge of how the disease impacts the patient's daily life.

Ginger.io ${ }^{5}$ is an application for mobile phones combined with a subscription service to manage depression, stress, and anxiety with the support of licensed therapists and psychiatrists. The application allows users to track their moods and collects data from sensors regarding activity levels, use of social networking

5 www.ginger.io 


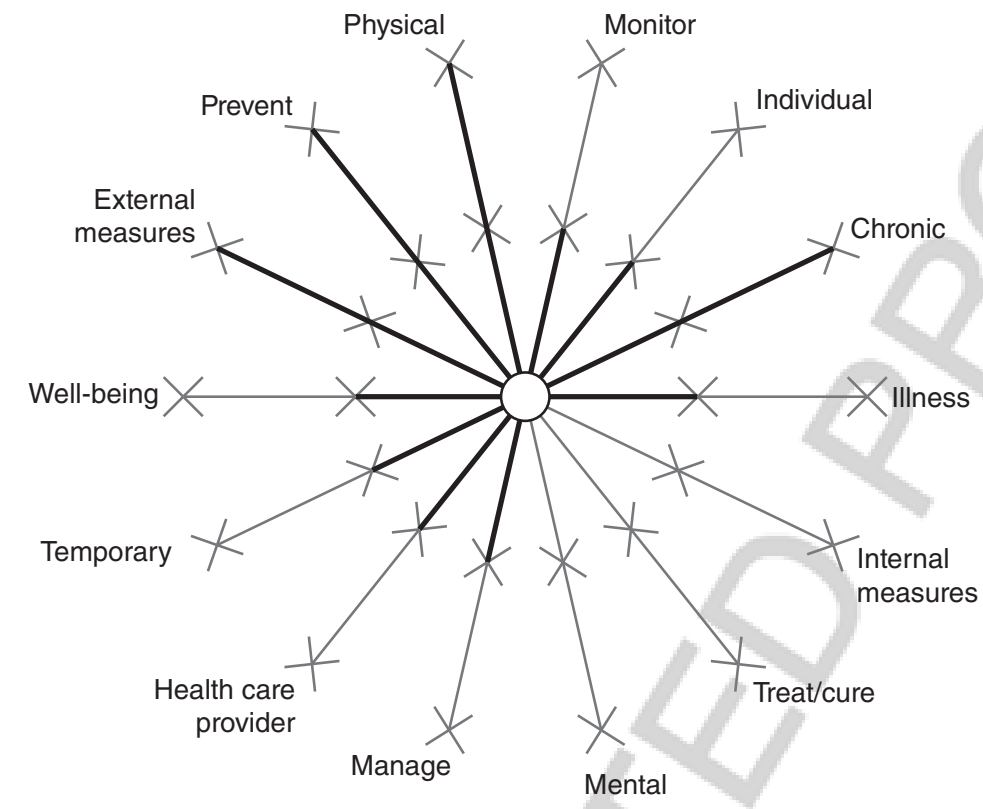

Figure 16.5 Propeller represented in the snowflake model.

applications, and phone use. Personalized counseling is provided via text and interactive video. In Figure 16.6, the dimensions of the Ginger.io are shown in the snowflake model.

Ginger.io is an app for mental illness. Depression, anxiety, and stress are temporary, but they have a tendency to come back if the person does not manage their well-being even when they are symptom free. The app in combination with support of the health care providers helps the individuals to manage and cure their stress, anxiety, and depression. The sensors are automatically collecting activity data generated by the person. Accordingly, the app collects internal measures, but it also collects external data like self-reported mood, phone calls, and social media activities. Also, through the app the users can communicate with health care providers about the data that has been recorded.

GPS SmartSole ${ }^{6}$ allows caregivers to use their smartphones or computers to track the movements of patients who are prone to wandering or getting lost due to Alzheimer's, dementia, brain injury, or other cognitive issues. The SmartSole looks like an ordinary shoe insert (such as those commonly used to provide additional cushioning or arch support) that can be placed in the patient's shoe. Sensors embedded into the insert allow for GPS tracking. In Figure 16.7, the dimensions of the SmartSole are shown in the snowflake model.

6 www.gpssmartsole.com 
16.4 Examples of IoT-Related Health Care Applications and Their Dimensions 463

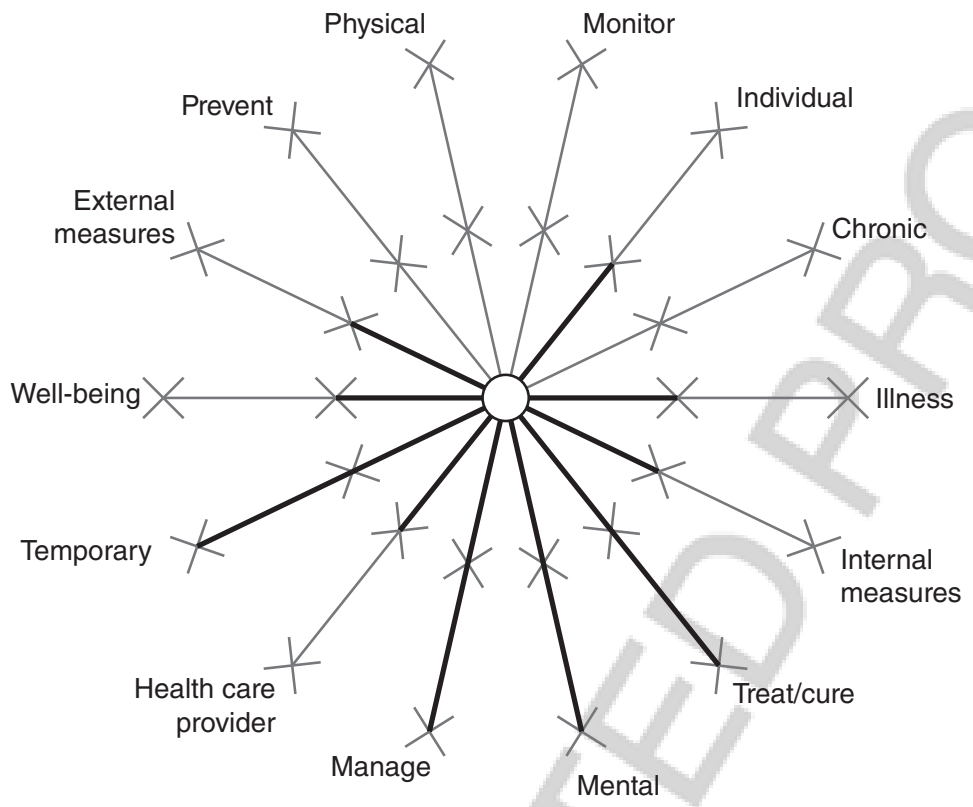

Figure 16.6 Ginger.io represented in the snowflake model.

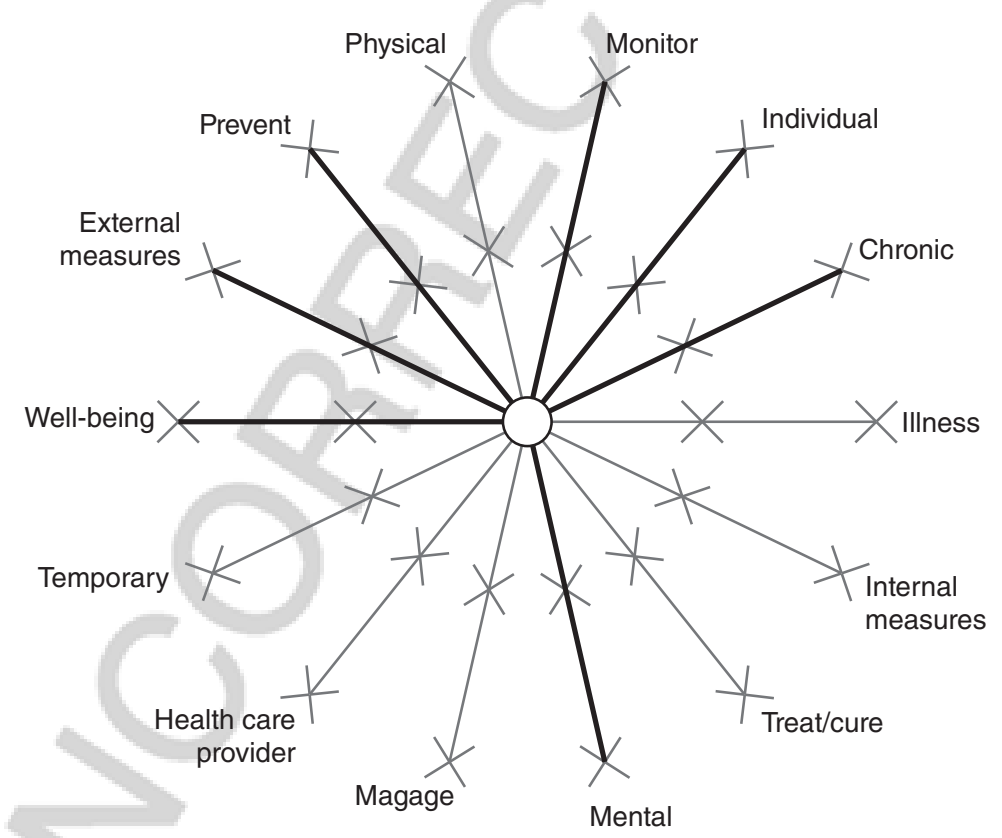

Figure 16.7 SmartSole represented in the snowflake model. 
GPS SmartSole is designed to empower people with cognitive impairments by giving them more independence because they can be given more freedom of movement, while at the same time keeping them safer because caregivers can track their location. Cognitive impairment is a chronic mental condition and GPS SmartSole supports those with such conditions to prevent them from the mental anguish of being lost and potentially from coming to harm. People with cognitive issues are often very dependent on significant others and GPS SmartSole makes it possible for the significant others to monitor and track the person's movements from a distance and make sure everything is as it should be. They can make sure the user has not moved outside the "safe zone," for example. GPS SafeSole can in this way promote well-being for both the person with cognitive impairments and their caretakers. The sole collects external data as it is equipped with a GPS sensor.

Philips Automated Medication Dispenser service ${ }^{7}$ uses a smart pill container that is programmed according to the patient's medication schedule and loaded with the appropriate medication by the caregiver. An alarm alerts patients that it is time to take their medication. At this time, the container will unlock and make the appropriate pills available. If pills are not taken on schedule, they will be stored inside the dispenser. If pills are missed repeatedly, the caregiver receives an alert. In Figure 16.8, the dimensions of the automated medical dispenser are shown in the snowflake model.

The automated medicine dispenser is designed for people who might forget to take their medicine. While the medicines dispensed could treat both physical and mental illness, its primary purpose is to serve as a memory aid, thus addressing a mental issue. The dispenser aims to help both the individual patient and the health care provider to manage the medicine intake. The device can be viewed both as preventive and as providing treatment as the reminders prevent the patients from forgetting to take the medicine that is essential for their treatment. The device also prevents the patient from taking more medicine than prescribed. It might be that the patient does not remember that she has taken the medicine and, therefore, takes it twice or more, with potentially serious consequences. To avoid this, the dispenser locks the compartments until it is time to take the new dose. The dispenser can be used by people with both temporary and chronic diseases. Forgetfulness itself can be temporary or permanent, although it is likely that the patient has an on-going problem with forgetfulness if the dispenser is prescribed by the health care provider. The automated medical dispenser is not only beneficial for the individual, but also for the health care provider as the nurse handling the medicine knows if the medicine is taken or not and it gives valuable information about further treatment. The medical dispenser is a stand-alone device, not connected to the user at all, meaning that it only involves external measurement. It does,

7 https://www.lifeline.philips.com/health-solutions/health-mdp.html 


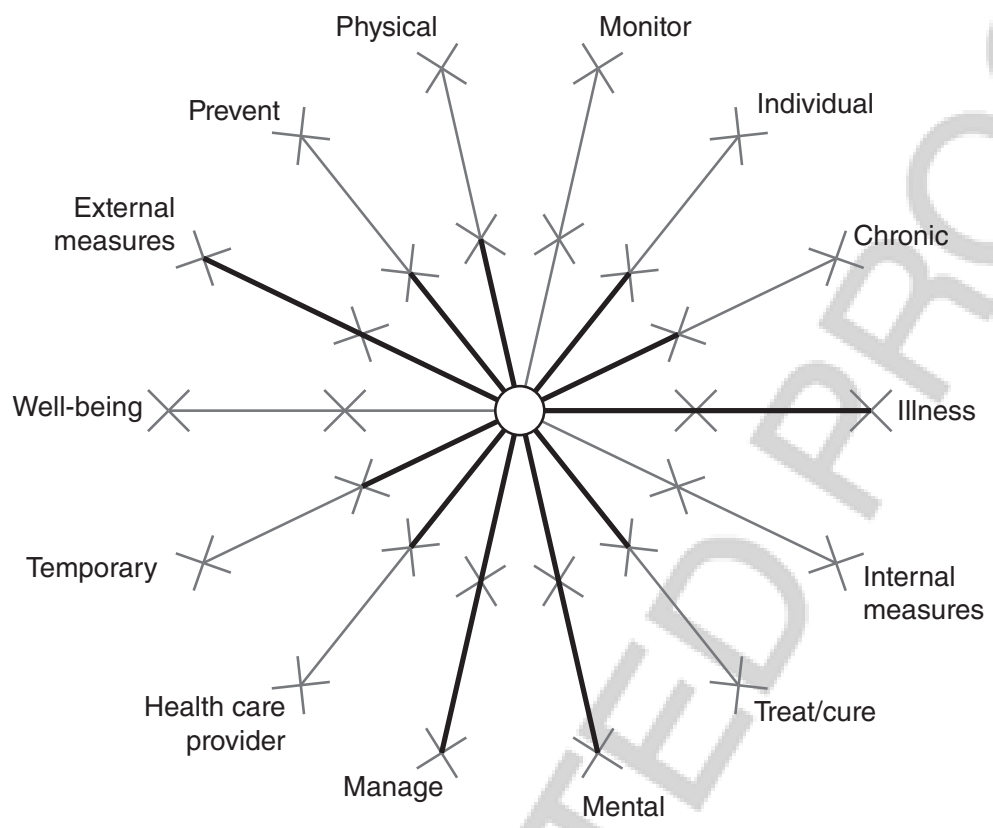

Figure 16.8 The automated medicine dispenser represented in the snowflake model.

however, communicate with other components of the IoT health care ecosystem. It alerts the patient according to a schedule when it is time to take a pill, and alerts the health care provider when the patient has missed taking a medication dose.

CoaguChe ${ }^{\circledR 8}$ monitors blood coagulation levels for patients who take bloodthinning drugs, such as warfarin, and sends the results to health care providers. The device is fed by a drop of blood and the display shows the INR-value (International Normalized Ratio), which indicates the blood's tendency to clot. The application also collects user comments regarding, for example, diet change, illness, missed medication, and travel, and patients can set reminders to check their blood. The data received by patients indicate trends and whether the blood coagulation levels are within a target range. In Figure 16.9, the dimensions of the CoaguCheck are shown in the snowflake model.

The CoaguCheck is designed for the individuals with an increased risk of blood clots and who take warfarin to keep the blood viscosity within a desired range. The accurate dose is established by measuring how long it takes for the blood to clot. The device's main objective is to make it possible for the patients to monitor the INR-value on their own without going to the hospital to take a blood sample. Instead of wasting time to travel to the hospital, the patient can call the

8 www.coaguchek.com 


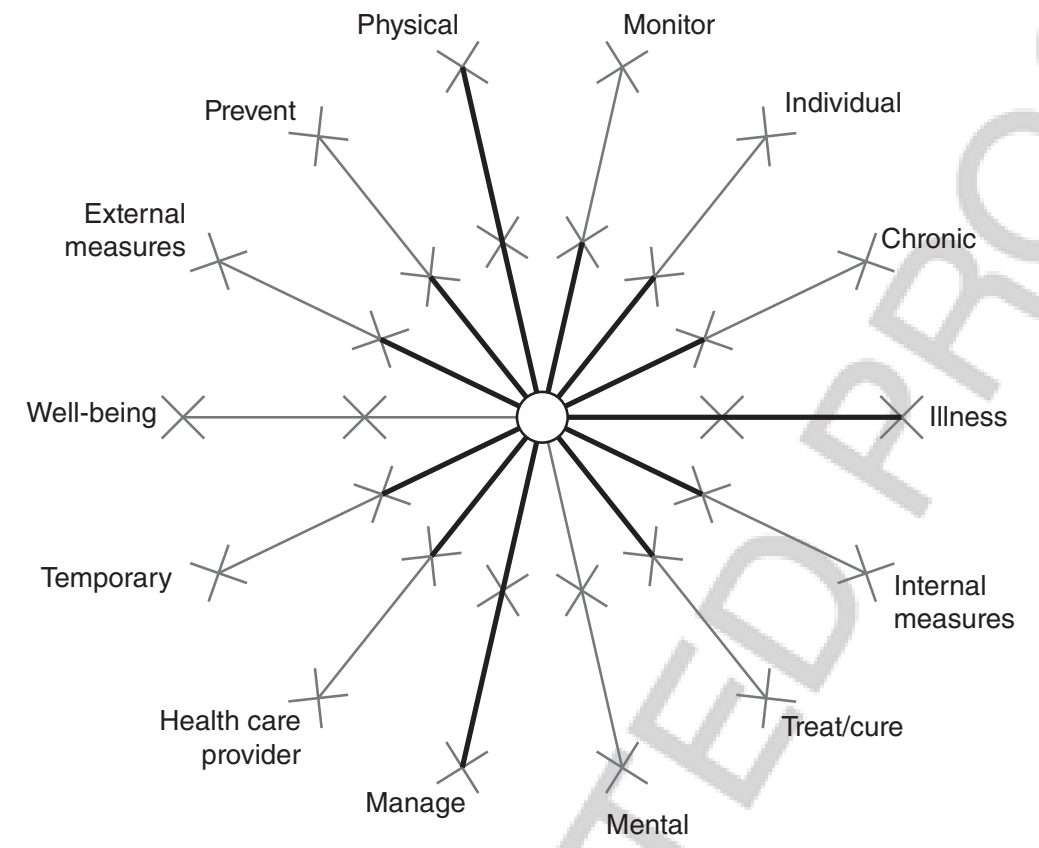

Figure 16.9 CoaguChek represented in the snowflake model.

health care provider and communicate the IRN-value and the health care provider can then change the dose if necessary. The self-measured INR-values can also help the patient to manage their value as they can learn how food and their lifestyle affect the INR-value. Measuring the INR-value often makes it possible to better adjust the treatment and also to prevent the values from being too low or too high because the dose can be changed more frequently. The reasons for taking warfarin can differ. Warfarin can be taken because the patient has had a blood clot. Only when and if the patient is determined not to have an increased risk for having more blood clots does he or she stop taking the medication. In this case, the warfarin is taken only temporarily, and accordingly, the CoaguCheck would be used only temporarily. But patients that have medical heart valve replacements need to be medicated with blood thinners like warfarin for the rest of their lives. The CoaguCheck does not only collect internal measurements such as the INR, but also external values the users report about, such as their diet.

An activity tracker for oncology treatment (a collaboration between Medidata ${ }^{9}$ and Memorial Sloan Kettering Cancer Center ${ }^{10}$ ) makes use of ordinary activity

9 https://www.mdsol.com/en/

10 https://www.mskcc.org/ 


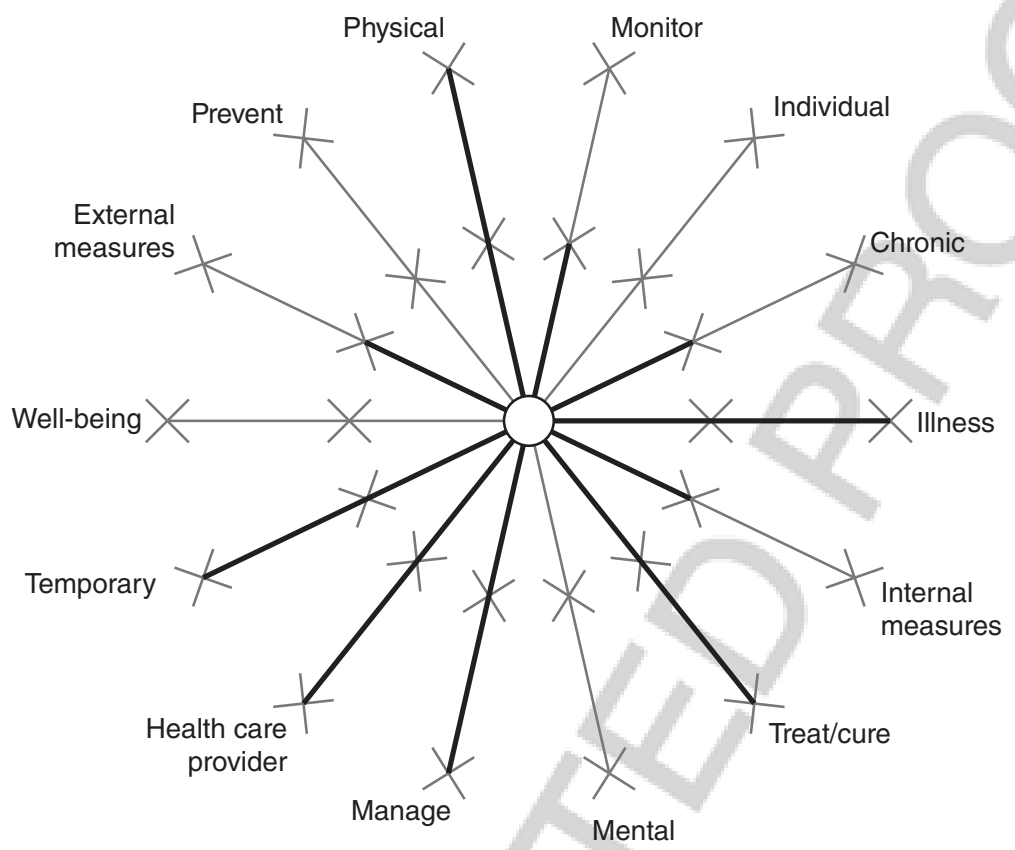

Figure 16.10 The activity tracker for oncology treatment represented in the snowflake model.

trackers on the market to allow health care professionals to measure the efficacy of drug treatment for multiple myeloma (a blood cancer). Wearable activity trackers and patient-reported data on health status enable medical researchers to evaluate the impact of drug treatments on patients. In Figure 16.10, the dimensions of the activity tracker for oncology treatment are shown in the snowflake model.

Today, multiple myeloma is not curable, but the survival rate has increased during the past decade due to improved treatment. The disease is physical and the patient has to live with it for the rest of their lives, but the illness is not classified as chronic and for that sake, it is shown as primarily temporary in the snowflake model. The activity tracker is used by the health care provider to manage the treatment and monitor the quality of the treatment as well as the patient's quality of life to better customize the treatment. As with the ordinary use of activity trackers, internal measurements like activity level and pulse rate are captured. In addition, the patients report their health status, so external values are also recorded.

Eversense continuous glucose monitoring ${ }^{11}$ systems enable diabetics to continuously measure their blood sugar level. A small cylinder-shaped sensor

11 https://eversensediabetes.com/cgm/ 


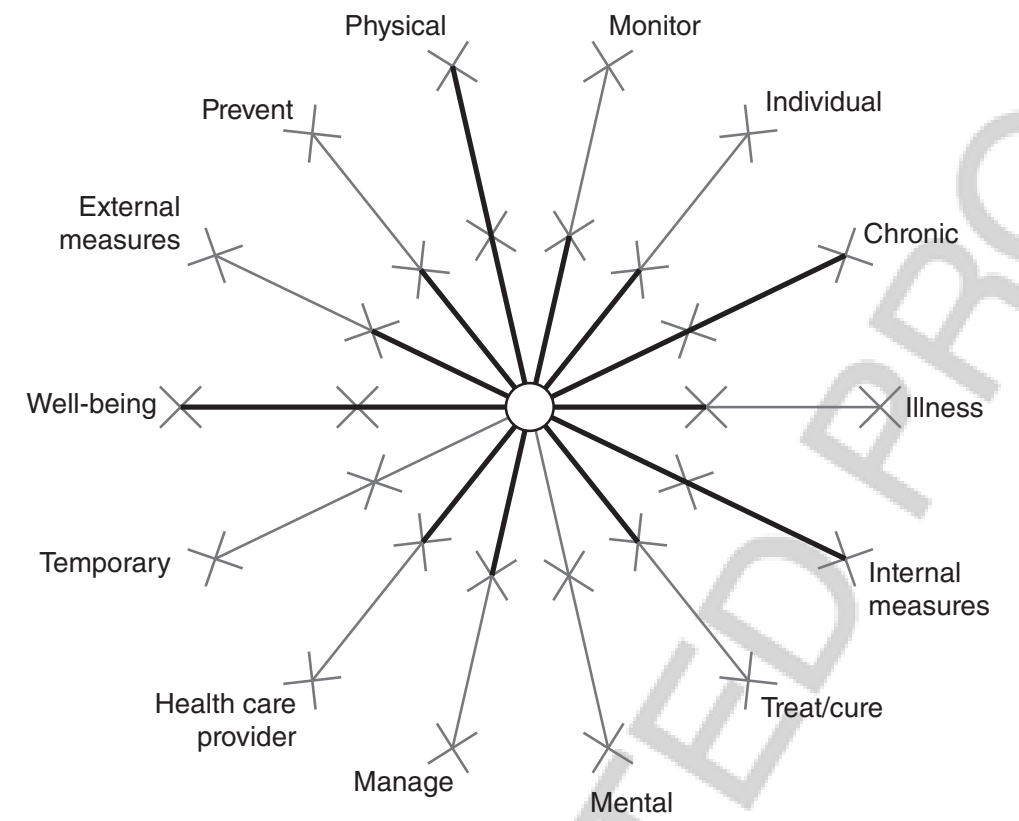

Figure 16.11 The continuous glucose monitoring meter represented in the snowflake model.

embedded under the skin measures levels of blood sugar in fluids in the tissues. A connected transmitter powers the sensor and sends readings wirelessly to a smartphone. The sensor does glucose readings every 5 minutes and the app can send predictive alerts before reaching a too low or high-glucose level. The app also allows the user to manually track events like meals, workouts, and insulin injections. Results can be shared with a health care provider. In Figure 16.11, the dimensions of the continuous glucose monitoring are shown in the snowflake model.

The continuous glucose monitoring system is designed for a chronic disease, diabetes. This is a physical disease the person has to live with throughout his or her life. For people with diabetes, it is important to both monitor and manage their blood glucose value. The patient has to monitor the glucose value to make sure the value is not too low or too high. If the values are not in a desirable range, the patient has to manage the blood glucose level in different ways. If the value is too low, the person has to eat something and if it is too high he or she needs to inject some insulin. To do that, the patient has to understand the monitored values and also know the appropriate action to take. The blood glucose monitoring system addresses the illness, but it is mainly used to make it possible for people to live a good life, for their well-being. While diabetes cannot yet be cured, those people with type 2 diabetes still have some insulin production of 
their own left and by monitoring and managing their blood glucose values and taking additional action they can actually treat their diabetes and prevent it from becoming worse. People with type 1 diabetes can try to prevent serious complications later on in life (damage to other organs and eyesight, for example) by keeping their glucose level in a normal range. The sensor itself only measures internal data-theblood glucosevalue-but the user can manually collect external datalike meals and insulin injections. The device mainly targets the individual but is also beneficial for the health care providers because the measurements and the trends in the measurements can help them support the patient by recommending insulin doses and actions to manage the blood glucose levels.

In summary, the types of health care applications using the IoT span a broad spectrum. The aforementioned applications focus on the patient, although the metrics and other data collected by some applications are more directly used by health care providers, family members, or other caregivers to monitor the patient. Some applications are quite comprehensive, in that they collect both biometric data from the patient's body as well as contextual data about the patient's environment and activities and feelings, while others focus on a very small set of metrics. Applications may help with the management or treatment of an illness or condition, or may simply allow monitoring of a patient's status on particular metrics. There are applications that support well-being and those that help to prevent illness or complications. By exploring these examples, the great potential for technology, particularly the IoT, to improve the quality of life for many patients becomes clear.

\subsection{Challenges}

Smart health care ecosystems are multidimensional systems of people, hardware, software, and networks operating in a complex legal and regulatory environment with potentially life or death consequences. Complicating this context are data and platform heterogeneity issues, a lack of comprehensive standards, and concerns with privacy, security, and accuracy of data. These systems have the potential to change the relationship between patients and their health care providers, as they impact the way health care providers perform their duties and the amount of responsibility patients may take in managing their own health. Each of these issues is discussed further.

\subsubsection{Lack of Standards}

The use of personal devices, such as smartphones and smart watches, sensors, and applications that provide data that can be used by both individual patients and health care providers, are relatively new developments in the technology and health care realms. It is not always entirely clear which regulatory bodies and 
agencies have jurisdiction over these applications, and to date (as is common), regulations have not kept up with technological developments (Vincent et al., 2015). While the International Standards Organization (ISO) has published international standards for medical equipment, these address safety and usability. Standards for connected medical devices and connected health software are currently under development.

Standards to ensure data compatibility and universal regulations regarding how data can be stored, transmitted, and used are also lacking. These are currently addressed regionally. In the United States, the Health Information Technology Standards Panel has been given this responsibility. A similar body, the Medicine and Health Care Products Regulatory Agency (MHRA) exists in the United Kingdom. However, for smart health care IoT applications to gain widespread use, global standards are needed. Without standards for platforms, functionality, communication interfaces, and data it is difficult to ensure the compatibility of different devices, sensors, and applications, particularly in a global context. This can slow both innovation as developers wait for standards to be set and adoption because certain applications may not be functional or legal in particular locations. In addition, it may not be possible to integrate the data generated by these smart health applications with existing digital medical records or to use them as input to hospital or clinical systems.

\subsubsection{Data Issues}

There are additional data issues beyond the lack of standards that present challenges in the health care environment. The types of data used by smart health systems may include personally identifiable information (PII) and healthrelated data that some patients might view as quite sensitive. As discussed in the previous chapters, IoT systems in general are known to have many security vulnerabilities, and these apply to the smart health care IoT ecosystem as well. Medical devices and consumer technologies often have lower levels of protection than do typical computer network devices. There have been many examples of hacking of medical and consumer devices connected to the Internet (e.g., Reel and Robertson, 2015), and these issues must be addressed to safeguard the personal and medical data saved and communicated via the IoT.

Another issue with data is the level of accuracy of the metrics produced by smartphones and wearable sensors. Comparisons of consumer devices such as step trackers have shown quite a bit of variation in the data captured by different devices (Guo et al., 2013). Data that is not quite accurate may not have significant impact for individuals who are tracking their steps, but if the inaccurate sensor is measuring blood glucose levels, for example, incorrect results could lead to serious consequences, including death of the patient. To account for this, most blood glucose monitoring devices require frequent (up to multiple times per day) calibration. The lack of accuracy causes some health care professionals to resist 
the use of smart health applications, although there have been reported examples of data from personal fitness trackers being used to support medical diagnosis. In one example, emergency room physicians used data from a patient's Fitbit to determine the appropriate treatment for a heart arrhythmia (Rudner et al., 2016).

Providing data to patients in a usable manner is another challenge. Patients have different levels of technological and medical literacy, and their ability to understand the meaning of health metrics will differ. The way in which data is filtered, summarized, and displayed can make a difference in how easily it is understood. Patients may have different preferences regarding how much data they receive, or how often they receive it. Therefore, smart health applications should take these individual differences into account and should enable some level of customization of the system.

\subsubsection{Changing the Health Care Provider-Patient Roles}

Smart health systems should not be viewed solely from a technological or medical perspective, but from a broader sociotechnical perspective that includes the impact of these systems on roles, relationships, organizations, and society in general. Building these applications requires not just technological competence, but also medical knowledge related to the particular specialty area addressed by the application. An understanding of human behavior, psychology, and motivation is needed to create systems that can assist patients in changing their behavior.

While smart health systems offer patients the opportunity to have more control of their health care, this comes with added responsibility. As patients take a more active role in managing their health, they have more duties in terms of monitoring various metrics, learning what the numbers and other visualizations mean and what actions to take, and possibly doing self-recording of data required by the application. This may be a welcome change for some patients, but for others this may be an unwanted burden.

These smart health systems may also impact the health care professional's role. Physicians and nurses may spend less time meeting with patients face-toface, and more time monitoring output from patient's devices and sensors. While this should enable health care professionals to provide better care to more patients, it may require more effort and offer less personal satisfaction for some practitioners. Just as the Internet has led to patient self-diagnosis (and often misdiagnosis), the volumes of data available to patients may also lead to some patients using more health care professionals' time as they request assistance in interpreting the data or seek treatment for nonserious issues.

\subsection{Conclusion}

Both advances in technology and increasing pressures for more effective and efficient methods of addressing health care issues have contributed to the 
growth in the number of applications of IoT in health care. Through the IoT, data captured by various sensors and devices can be shared with applications used by patients and health care providers to monitor physiological metrics and other data that can be used in the management, treatment, or prevention of various illnesses or other medical conditions. Both physical and mental conditions are addressed by these systems. Some systems focus on well-being and quality of life issues as well. This chapter presented a people-focused view on IoT by providing an overview of the components that may be included in an IoT-based smart health ecosystem and introduced a set of dimensions to consider in smart health applications. In summary, there are many challenges facing the widespread adoption of smart IoT health care applications. However, for many of these applications, the benefits outweigh the risks, and therefore this area is expected to continue to grow at a rapid pace.

Future developments will include the integration of artificial intelligence components to supplement or take the place of the health care professional or other caregiver or the social support agents. Miniaturization (nanotechnology) will enable injectable sensors to become common. Injectable and implanted devices-embedded chips and other sensors to monitor physiological status - are beginning to be used, as are electronic patches and electronic tattoos that serve as sensors (Motti and Caine, 2015). Augmented reality will also be used in smart health care applications both to aid health care professionals in integrating data on patients, conditions, and treatments and to assist patients in using the data collected by these systems to manage their health. Innovations in power consumption (increasing battery life and generating power through the user's movements, for example) and increased bandwidth will allow wearable devices to last longer and to transmit more data more quickly. In addition to advances in sensor technology, user interfaces, and computational power, we can also expect to see more integration across platforms, so that patients can monitor multiple aspects of their health simultaneously thus allowing a more holistic perspective not just addressing one condition or symptom, but instead addressing the broader context of patients dealing with multiple health-related issues. As smart health IoT systems become more accurate, powerful, and reliable and legal and regulatory systems are updated to include these systems, they will become integrated into the health care infrastructure.

\section{Acknowledgments}

This work has been carried out within the research profile "Internet of Things and People", funded by the Knowledge Foundation and Malmö University in collaboration with 10 industrial partners. 


\section{References}

Glaros, C. and Fotiadis, D.I. (2005) Wearable devices in healthcare. Studies in Fuzziness and Soft Computing, 184, 237-264.

Guo, F., Li, Y., Kankanhalli, M.S., and Brown, M.S. (2013) An evaluation of wearable activity monitoring devices. Proceedings of the 1st ACM International Workshop on Personal Data Meets Distributed Multimedia (PDM'13), October 22, 2013, Barcelona, Spain. pp. 31-34.

Motti, V.G. and Caine, K. (2015) An Overview of Wearable Applications for Healthcare: Requirements and Challenges, UBICOMP/ISWC '15 Adjunct, September 07-11, 2015, Osaka, Japan, pp. 635-641.

Reel, M. and Robertson, J. (2015) Hospital gear could save your life or hack your identity. Bloomberg Businessweek, November 2015. Available at https://www .bloomberg.com/features/2015-hospital-hack/ on 05/01/17.

Rudner, J., McDougall, C., Sailam, V., Smith, M., and Sacchetti, A. (2016) Interrogation of patient smartphone activity tracker to assist arrhythmia management. Annals of Emergency Medicine, 68(3), 292-294.

United Nations (2010) The Millennium Development Goals Report 2010. Available at: http://www.un.org/millenniumgoals/pdf/MDG\%20Report\% 202010\%20En\%20r15\%20-low\%20res\%2020100615\%20-.pdf (accessed March 03, 2017).

Vincent, C.J., Niezen, G., O'Kane, A.A., and Stawarz, K. (2015) Can standards and regulations keep up with health technology? JMIR mhealth uhealth, 3(2), e64.

WHO (2010) Health Systems Financing: The Path to Universal Coverage, World Health Organization (WHO). 
